Sociologie et sociétés

\title{
Le parcours vers le premier enfant dans les régimes providentiels
}

Une analyse séquentielle des trajectoires des femmes en Italie, en Suède et en France

\section{The Journey to the First Child in Welfare Regimes}

Sequence Analysis of the Trajectories of Women in Italy,

\section{Sweden and France}

\section{Laetitia Koenig}

Volume 45, numéro 1, printemps 2013

Inégalités, parcours de vie et politiques publiques

Inequality, Life Course and Public Policy

URI : https://id.erudit.org/iderudit/1016402ar

DOI : https://doi.org/10.7202/1016402ar

Aller au sommaire du numéro

Éditeur(s)

Les Presses de l'Université de Montréal

ISSN

0038-030X (imprimé)

1492-1375 (numérique)

Découvrir la revue

Citer cet article

Koenig, L. (2013). Le parcours vers le premier enfant dans les régimes providentiels : une analyse séquentielle des trajectoires des femmes en Italie, en Suède et en France. Sociologie et sociétés, 45(1), 229-254.

https://doi.org/10.7202/1016402ar 


\section{Le parcours vers le premier enfant dans les régimes providentiels}

Une analyse séquentielle des trajectoires des femmes en Italie, en Suède et en France

\section{LAETITIA KOENIG}

Département de sociologie Université de Montréal C. P. 6128 , succursale Centre-ville Montréal (Québec) $\mathrm{H}_{3} \mathrm{C}_{3} / 7$

Courriel: laetitia.koenig@gmail.com

$\mathrm{L}$ ES SOCIÉTÉS OCCIDENTALES connaissent presque toutes, depuis au moins quelques décennies, un problème de baisse marquée de la fécondité. Les naissances ne parviennent plus à assurer le renouvellement de la population. Cette chute transforme en profondeur la distribution par âge de la population, où les personnes âgées représentent un poids de plus en plus important. Pour apporter un éclairage sur cette importante question d'intérêt public, nous avons voulu comparer quelques pays quant au parcours qui conduit, ou non, à la naissance d'un premier enfant. La proportion de femmes et de couples qui franchiront cette étape est évidemment déterminante pour le niveau de fécondité, directement, de même qu'en tant qu'étape vers la venue des enfants subséquents.

Pour comprendre ce parcours vers le premier enfant il faut, dans la plupart des cas, mettre l'accent sur la population des jeunes. Or la jeunesse est en pleine transformation, comme le montrent par exemple les travaux d'Olivier Galland (2000 et 2007). Les séquences habituelles entre les étapes d'entrée dans la vie adulte ne sont plus les mêmes, ou en tout cas s'éloignent de la trajectoire plus classique qui prévalait il y a quelques décennies: fin des études, entrée dans le marché du travail, mariage, naissance du premier enfant (Galland, 2000 et 2007; Myles, 2005). L'allongement des études, devenu 
une condition de succès dans l'univers de l'emploi, de même que les changements culturels des rapports entre générations et entre genres ont bouleversé, dans plusieurs pays, les conditions d'entrée dans la vie adulte et en particulier de l'accès à des conditions jugées favorables à la venue d'un premier enfant. La formation d'un couple demeure certes la situation où se produit l'immense majorité des premières naissances; mais elle n'est pas une condition suffisante, tout juste une condition quasi nécessaire. Plus encore, les couples sont bien moins souvent mariés que ce n'était le cas il y a quelques décennies, et ils sont plus instables (Monnier, 2006).

En fait, nous arguerons que la venue d'un premier enfant repose sur la capacité des individus à réunir un certain nombre de conditions pour faire face aux besoins que génère une naissance. Selon Toulemon, les individus incluent les enfants dans leur parcours de vie en s'assurant que ces conditions resteront au moins semblables, voire iront en s'améliorant (1994). Les conditions socio-économiques des individus sont, comme le montrent de nombreux observateurs, importantes dans la création d'une famille, puisqu'elles offrent une certaine sécurité, phénomène encadrant le futur de la nouvelle cellule familiale (Majnomi-D'intignano, 2004). L'investissement de temps est également nécessaire pour encadrer une naissance et pose la question de l'accessibilité aux services de garde publics et privés, des ressources nécessaires pour l'éducation, etc. (Billari, 2004). Les femmes et les couples vivent dans un contexte particulier leur permettant d'obtenir un certain nombre de soutiens pouvant leur faciliter l'inscription d'un enfant dans leur parcours de vie (Cohu et al., 2005; Njoki, 2006). Selon EspingAndersen, initiateur de la typologie des régimes providentiels, les soutiens sont variables d'un pays et d'un régime providentiel à l'autre et répondent différemment, indirectement ou directement, à des besoins spécifiques (1999).

Cette recherche originale suggère, sous l'égide de la perspective des parcours de vie présenté par Bernard (2007) et de la typologie des régimes providentiels selon Esping-Andersen (1999) et ses critiques, de comprendre les cheminements qu'empruntent les femmes avant la naissance de leur premier enfant. L'analyse séquentielle, proposée par les travaux de Rajulton et le programme Lifehist, a été utilisée car elle identifie différemment les comportements des individus en tenant compte de l'enchầnement de différentes séquences et apporte une approche différente de la compréhension des comportements en matière de fécondité des femmes (1999).

Nous proposons ainsi une comparaison de cheminements vers la première maternité présents dans trois types de régimes providentiels identifiés par la littérature ayant un accès différent aux ressources requises, de même qu'un certain nombre de contraintes (Gauthier, 2002). Il est de fait question de confirmer l'existence de trois modèles de cheminement vers la première maternité propres à chacun de ces régimes (et leur résilience ou non à travers le temps durant la période observée) à travers les évènements du passage à l'âge adulte tout en montrant la nécessité de tenir compte des contextes sociétaux dans la compréhension du phénomène de la fécondité (Cavalli, 2003). 


\section{PARCOURS DE VIE ET RÉGIMES PROVIDENTIELS}

Cette recherche se fonde sur la perspective des parcours de vie, selon Bernard, qui prend en compte la dimension contextuelle des vies des individus (2007): si les parcours de vie se diversifient, s'ils sont propres à chaque individu et s'ils comportent de multiples facettes, ils s'inscrivent également dans des milieux socialement construits. La décision de fonder une famille appartient aux individus, qui, selon Elder, s'inscrivent dans le temps individuel, générationnel et historique (1998), et qui est prise selon Bernard dans un contexte précis offrant un cadre culturel et institutionnel déterminant la mobilisation des ressources selon certaines occasions et contraintes. À l'instar des politiques démarchandisantes ${ }^{1}$, Mathieu a montré que les régimes providentiels offrent également différents mécanismes de soutien à la mobilisation des ressources des jeunes à travers des politiques défamilialisantes ${ }^{2}$ (2003). Chacun des régimes providentiels se démarque par une logique propre et influe incontestablement sur les parcours de vie des individus en faisant partie.

La typologie d'Esping-Andersen présente le régime social-démocrate suédois ayant pour base le principe d'égalité où l'État y constitue un vecteur particulièrement important de bien-être, en cherchant à réduire les inégalités sociales et économiques. Il s'agit d'une perspective basée sur l'universalisme des droits et une redistribution des ressources aux individus. Les mesures et les politiques sont nombreuses et permettent une forte démarchandisation des femmes et des hommes, en particulier quand les obligations familiales l'exigent. Les recherches ultérieures de Mathieu (2003) montrent que la formation du capital humain y était privilégiée, les services de garde permettant aux parents de demeurer sur le marché du travail et les politiques défamilialisantes d'obtenir des congés parentaux en conservant la quasi-totalité du revenu avant de se réinsérer en emploi sans perdre les avantages acquis auparavant. Ces aménagements permettaient aux femmes de déployer leurs ambitions de carrière et favorisaient grandement la fondation d'une famille (Marc et al., 2005). Les pères, à travers le congé parental et le congé paternel, étaient également en mesure de s'impliquer dans l'éducation et les soins apportés aux enfants. Véritable régime de "rôle partagé», le régime social-démocrate contribue à la promotion de l'égalité entre les sexes.

Le régime conservateur français, quant à lui, présentait des assurances sociales obligatoires procurant une redistribution basée sur les contributions passées, définies selon la catégorie socioprofessionnelle, et la situation familiale (Esping-Andersen, 1999). Il offrait alors un système de protection sociale mettant temporairement les travailleurs et leurs familles à l'abri des principaux risques sociaux pouvant les affecter. La forte volonté de maintenir la cohésion et l'intégration sociale produisait, à travers le principe de solidarité, un certain nombre de mesures sociales et économiques dans

1. La démarchandisation ( «decommodification») caractérise la relation entre deux principaux piliers de bien-être: l'État et le marché, mesure le degré d'assistance de l'État envers les individus en ce qui concerne leur indépendance face au marché du travail (ensemble de droits, de transferts et de services).

2. La défamilialisation correspond aux mécanismes mis en place par l'État afin de collectiviser certains coûts économiques et sociaux reliés à la venue d'un enfant par exemple. 
ce pays: intervention généreuse de l'État en matière d'allocations pour les familles (Croisetière, 2006) et les droits de démarchandisation octroyaient la possibilité aux individus d'être indépendants du marché du travail (Nicole-Drancourt, 2006). Toutefois, c'est le réseau familial qui était alors le moyen privilégié pour alléger les charges reliées à la venue des enfants quand les parents travaillaient (les équipements d'accueil pour les enfants étant insuffisants) ou en cas de conjoncture économique difficile, car les mesures défamilialisantes y étaient très faibles. Par ailleurs, la dynamique des relations de genre générait une répartition sexuée des rôles dans la famille (Mathieu, 2003). L'accès au marché du travail était plus restreint pour les femmes (Dufour-Kippelen, 2001; Mathieu parle d'un régime d'«emploi restreint», 2003) et compromettait l'épanouissement de leur carrière. L'occupation d'un emploi atypique et ayant peu de flexibilité était courant pour les femmes et mères dans ce modèle (Pinelli et al., 2001).

Le régime familialiste italien, classé par la typologie d'Esping-Andersen avec les pays conservateurs, était pourtant plus rudimentaire avec un fort attachement aux valeurs religieuses plus marqué que le régime conservateur classique. Plus traditionaliste, ce quatrième régime regroupe les pays méditerranéens qui émanent de nombreuses critiques à la typologie d'Esping-Andersen (Ferrara, 1996; Mathieu, 2003). Dans ce régime, les droits de défamilialisation (la collectivisation partielle des coûts et des contraintes reliés à la prise en charge et à l'éducation des enfants) étaient encore plus faibles: la fonction de soins était à la charge des individus et des familles. Les femmes étaient assez touchées par le faible apport de l'État puisque cela diminuait leurs possibilités d'être indépendantes de l'institution familiale et de participer au marché du travail (Palomba, 2001), les confinant davantage à la sphère domestique (Cooke, 2006). Il y avait une très forte dichotomie entre les sexes et la répartition des rôles dans la sphère professionnelle et privée qui présente des structures familiales très traditionnelles (Cassese, 2001; Livi Bacci et Salvini, 2000). En outre, l'État italien encourageait, à travers les droits de démarchandisation, une importante séparation des rôles entre les genres dans la société quant à l'accessibilité à l'éducation et au marché du travail (Mathieu, 2003; Mc Donald, 1997). Enfin, les solidarités intergénérationnelles permettaient parfois de compenser le peu de prise en charge des enfants par les régimes traditionalistes, du moins lorsque la mobilité géographique des cohortes les plus jeunes n'était pas un frein (Cicchelli et al., 2007).

\section{DON NÉES ET MÉTHODE}

Pour répondre à la fois à nos ambitions de capter les comportements de fécondité des femmes en matière d'articulation des différentes aspirations dans leur vie, et à la fois de tenir compte de la manière dont ces comportements s'inscrivent dans les différents régimes providentiels sélectionnés, nous avons choisi d'utiliser les données récoltées dans les années 1990 par les enquêtes sur la famille en Suède, en Italie et en France (FFS, Fecondity and Fertility Survey). Ce projet unique n'avait pas seulement l'ambition de collecter de nouvelles données sur la fécondité et la famille en suivant le modèle 


\section{L'état de la fécondité et de l'emploi des femmes en Italie, en Suède et en France}

Dans les pays occidentaux et durant la période d'observation, ce sont surtout les pays du sud de l'Europe qui connaissent un fort recul du nombre d'enfants par famille. L'Italie affiche une chute draconienne des taux de fécondité avec 2,42 en 1970 à 1,22 enfants par femme en 1994. Le contexte familial dans lequel les naissances se produisent est tout aussi intéressant, alors qu'en 1970 seulement 2,2\% des naissances survenaient hors du cadre du mariage, en 1994 ce pourcentage n'avait guère évolué puisqu'alors 7,3 \% des naissances se produisaient hors mariage. Si le modèle culturel familialiste semble survivre, mais difficilement; les besoins économiques ne sont pas comblés par l'entrée des femmes sur le marché du travail, puisque relativement peu de femmes durant la période observée travaillent en Italie et leur accession au marché du travail est sensiblement freinée comparativement aux autres pays occidentaux. Ainsi, en 1970 la société italienne intégrait 35,1 \% des femmes en âge de travailler sur le marché du travail et en 1994 la participation n'avait guère augmenté puisque seulement 40,2 \% des femmes exerçaient une activité sur le marché du travail (Pinelli et al., 2001).

La Suède est un des rares pays affichant des taux de fécondité élevés et relativement stables à travers le temps: ils se sont maintenus, passant de 1,92 en 1970 à 1,89 enfant par femme en 1994. De plus, la stabilité de la fécondité suédoise à travers le temps est intéressante compte tenu de la diminution importante des naissances dans le cadre du mariage (18,4\% en 1970 des naissances se produisaient hors mariage, contre 51,6 \% en 1994). La part des Suédoises participantes au marché du travail est considérable et en constante évolution: en 1970 la société suédoise intégrait déjà 52,5 \% des femmes en âge de travailler sur le marché du travail et en 1994 cette part atteignait 74,0 \% (Pinelli et al., 2001).

La France présente des taux de fécondité déclinants mais assez élevés depuis les dernières décennies: 2,47 en 1970 à 1,65 en 1994 avec une augmentation progressive des naissances hors mariage (de 6,8 \% en 1970 à 36,1\% en 1994). Par ailleurs, elle conserve des aspects culturels très familialistes et développe parallèlement des politiques sociales et familiales permettant, en particulier des ajustements sur marché du travail. Cela se traduit par des taux de participation au marché du travail relativement élevés avec en 1970, $49,7 \%$ des femmes en âge de travailler intégrées au marché du travail et 57,6 \% en 1994 (Pinelli et al., 2001). 
de ces prédécesseurs, mais également celle d'élargir les perspectives de la recherche en fécondité en tenant compte des multiples facettes du comportement des individus. Au-delà de l'identification des comportements de fécondité et des intentions des individus, il visait la compréhension de la fécondité et des comportements familiaux à travers le temps en collectant des données rétrospectives sur les histoires individuelles à travers plusieurs dimensions importantes des vies des répondants (matrimoniale, professionnelle, scolaire, migratoire) (Festy et al., 2002).

Pour définir les différents cheminements dans les régimes providentiels sélectionnés, nous avons choisi d'utiliser ces données dans la mesure où il s'agit des dernières disponibles présentant les deux caractéristiques nous étant nécessaires, sont la dimension biographique de la collecte et la présence de données standardisées pour permettre la comparaison. Ces deux principales forces du projet FFS n'ont pas été retrouvées depuis puisqu'aucune mise à jour ou autre projet d'une telle ampleur n'ont pour le moment été réalisés. La dimension exploratoire de cette recherche quant à la construction de trajectoires de type séquentiel tempère cette relative ancienneté des données puisqu'il est davantage question de reconstruire l'enchaînement des évènements formant des trajectoires types présentes dans trois différents régimes providentiels que de présenter des relations causales inexplorées en termes de fécondité en Europe (Abbott, 1995).

Si le choix de ces données nous apparaît le plus pertinent pour réaliser les trajectoires, il est nécessaire d'apporter quelques nuances pour relativiser nos résultats. En premier lieu, le projet FFS a privilégié quant à la collecte des données une totale autonomie de la part des pays participants; en conséquence, celle-ci n’a pas été réalisée au même moment dans les trois pays sélectionnés. Si onze années séparent la collecte de données du premier pays participant au projet et celle du dernier pays et que ce délai est assez long, compte tenu des changements rapides de la sphère familiale à ce moment-ci (Festy et al., 2002), l'écart est, toutefois, assez réduit en ce qui concerne les trois pays de notre recherche (1992 à 1996). Cette différence dans les périodes de collecte ne contrecarre pas nos analyses, dans la mesure où les trois régimes que nous étudions conservent les mêmes tendances sociales, économiques et politiques et surtout suivent la même logique, au cours des dernières décennies, dans leurs objectifs de soutien aux familles, aux travailleurs, etc. Surtout, nous nous ne nous intéressons pas à l'impact de politiques spécifiques, qui ont pu être mises en place, sur les trajectoires vers la fondation de la famille, mais davantage aux différences globales de trajectoires des femmes dans trois régimes providentiels distincts.

En deuxième lieu et si, comme le soulignent Courgeau et Lelièvre (1989), il est important dans l'analyse des enquêtes rétrospectives de tenir compte de la sélectivité de l'échantillon, qui ne reflète pas intégralement la population à cause de l'absence de certains individus au moment de l'enquête (migration et décès), nous devons préciser que les bases de données en notre possession ne contenaient pas de pondération permettant d'établir la représentativité des échantillons (par rapport aux taux de nonréponses). Sans présenter en détail la structure des non-réponses établie dans les 


\section{Le projet FFS en Italie, en Suède et en France}

L'enquête italienne a été réalisée entre le mois de novembre 1995 et le mois de janvier 1996 par une agence privée ( Metron» à Rome), étroitement encadrée par Istat (Institut national de la statistique italien). Le fichier standard de l'enquête regroupe de l'information concernant 6030 répondants âgés de 20 à 49 ans (4824 femmes et 1206 hommes), soit des répondants nés entre 1946 et 1975. En Italie, la collecte des données a été réalisée grâce à un échantillonnage par grappe à trois niveaux (municipalité, registres électoraux et l'âge). La stratification d'échantillonnage, en tenant compte de la dimension géographique, est intéressante dans le cas de l'Italie compte tenu des fortes différences économiques, sociales et culturelles au sein des différentes régions. À titre d'exemple, le niveau de fécondité est pratiquement le double dans les régions du Sud par rapport aux régions du Nord (De Simoni, 1990).

Le projet FFS en Suède a été réalisé de la fin de 1992 au début de 1993 par Statistique Suède. Cette enquête a permis de collecter des données auprès de 4984 répondants âgés de 23, 28, 33, 38 et 43 ans (3318 femmes et 1666 hommes), soit des répondants nés en 1949, 1954, 1959, 1964 et 1969. Le plan de sondage suédois, conduit sous l'appellation de l'Enquête suédoise sur la famille, répond directement aux critères mis en place par le projet FFS puisque l'échantillon a été réalisé par une stratification aléatoire où l'individu est l'unité d'échantillonnage et où l'année de naissance et le sexe sont les variables de stratification. Cette méthode permet d'élaborer un échantillon directement utilisable, pour les analyses de parcours de vie et d'identifier dans le cas échéant les effets de politiques spécifiques.

L'enquête française a été conduite par l'INSEE (Institut national de la statistique et des études économiques) en avril et en mai 1994. Le fichier de données standard de l'enquête présente des informations concernant 4885 répondants âgés de 20 à 49 ans (2944 femmes et 1941 hommes), soit des répondants nés entre 1944 et 1973 . La collecte de données a été réalisée à la suite de l'enquête sur l'emploi. Cette enquête nationale et annuelle concerne 75000 ménages, elle est de type longitudinal prospectif et cible tous les adultes de 15 ans et plus constituant le ménage. Un seul répondant adulte, dans chaque ménage, était sélectionné par la méthode KISH (sélection aléatoire d'un individu au sein du ménage échantillonné) pour répondre à l'enquête FFS. 
rapports nationaux du projet FFS, certains éléments sont à relever ici pour permettre d'évaluer cette représentativité. Selon l'analyse des rapports nationaux faite par Festy et al. (2002), des différences significatives quant à la provenance géographique (certaines régions à forte densité de population affichent des taux de non-réponses plus élevés), l'âge des répondantes (taux plus importants dans les groupes d'âge plus élevé) et leur statut matrimonial (auprès des femmes non mariées) ont été identifiées. En outre, s'il est difficile de quantifier la sous-représentation des célibataires, on s'attend à ce que cela affecte minimalement nos résultats dans la mesure où nous nous intéressons au parcours jusqu'au premier enfant qui nécessite généralement un cheminement de mise en couple et un historique matrimonial/conjugal; or les célibataires au moment de l'enquête ne s'insèrent pas dans l'appréhension directe du phénomène que nous cherchons à étudier, mais plutôt d'une manière indirecte. Ainsi, les femmes qui ne sont pas mariées ont plus tendance à présenter des trajectoires diversifiées avec de multiples séquences conjugales; leur sous-représentativité pourrait conduire à sousestimer la diversité des trajectoires vécues, ce qui ne pourrait que limiter les différences que nous observons, notre interprétation nous apparaît donc vraie a fortiori. Par ailleurs, les taux de non-réponses des non-mariées sont d'autant plus forts que les femmes sont âgées; cela affecte encore moins nos conclusions dans la mesure où les femmes plus âgées ont une probabilité plus faible de ne pas être en couple (elles sont rares dans la population, ce qui est par ailleurs vérifiable par l'état civil pratiquement identique dans les trois pays). Enfin, plusieurs stratégies utilisées, par exemple dans la réalisation du plan de sondage, ont permis de contrer les biais liés à la non-réponse. Comme cela a été le cas dans la collecte française, qui était basée sur la situation matrimoniale lors de la construction de l'échantillon.

En dernier lieu, un problème touchant la plupart des collectes des pays participants au projet FFS est l'autonomie relative et permise pour la constitution du questionnaire (d'autant plus que certains pays, comme la France, ont adapté des enquêtes nationales déjà existantes pour répondre à ce projet, où la conservation d'une continuité historique a primé sur le questionnaire modèle proposé par le PAU). En outre, Festy et al. (2002) ont relevé certaines ambiguïtés quant à la définition des termes et des concepts employés dans le questionnaire. Ce que nous verrons plus loin dans la présentation des évènements constituant l'analyse.

Nous avons sélectionné l'ensemble des femmes échantillonnées présentes dans les trois fichiers de données qui correspondent aux enquêtes italienne, suédoise et française. Par choix méthodologique, nous avons conservé dans la construction et l'analyse des trajectoires, les femmes n'ayant pas ou pas encore eu d'enfant: la contribution de ces dernières est très importante sur le plan interprétatif et elle est essentielle au calcul des probabilités de trajectoires. En outre, pour atteindre l'objectif qui est de comparer, à travers le temps, les trajectoires des femmes vers la première maternité, nous avons séparé chacun de ces échantillons en deux sous-échantillons identifiant deux cohortes par pays: les femmes ayant plus de 35 ans et les femmes ayant moins de 35 ans au moment de l'enquête. Si les périodes couvertes par les trois pays ne sont pas totalement 
identiques, l'analyse nous permet d'identifier une période commune aux trois pays s'étalant de 1960 (année où les femmes les plus âgées interrogées ont 15 ans) à 1990 (année où les femmes les plus jeunes ont au moins 15 ans) ${ }^{3}$. Concrètement, ces données nous permettront de comparer différents modèles de cheminement vers la première maternité et d'identifier de quelle manière ils évoluent. Sans chercher à relier systématiquement la mise en place de nouvelles politiques familiales et les comportements de fécondité, la constitution de ces trajectoires vers la première maternité permet d'entrevoir les modes d'accès privilégiés selon le régime providentiel et de présenter trois types d'évolution des modèles de cheminement vers la première maternité: ainsi les modèles, à travers la période 1960-1990, peuvent se reproduire, s'établir plus fermement ou au contraire changer.

En vue de dresser les trajectoires types des femmes menant au premier enfant, nous avons choisi d'utiliser la méthode originale, développée par Rajulton (1999), sous le nom de «state space approach». Elle offre la possibilité de décrire le devenir des femmes à partir de différents états. De fait, elle nous permet de répondre à nos questionnements en établissant l'intensité et le calendrier des transitions vécues. La fécondité peut grâce à cette méthode être envisagée comme une transition de «nonmère » à "mère » en tenant compte de l'enchaînement de plusieurs évènements intermédiaires. Cette méthode repose directement sur le postulat que les transitions passées, d'un statut à l'autre, déterminent les transitions suivantes qui vont être vécues. Elle diffère nettement de l'analyse biographique, qui met davantage l'accent sur la durée ou le rythme entre les évènements concernés et les premières naissances (Billari, 2006), en construisant des trajectoires qui se concentrent sur l'enchaînement des évènements. Sous l'œil de la perspective des parcours de vie, la méthode choisie favorise la construction de trajectoires par l'agencement séquentiel des évènements vers la première maternité. Par conséquent, c'est l'analyse de l'agencement des évènements concernés qui est au centre de cette recherche.

L'application pratique de la méthode aux données de l'enquête FFS a été réalisée en utilisant le programme informatique indépendant "Lifehist», conceptualisé et réalisé par Rajulton (1992 et 1999). Le programme trace l'enchaînement des évènements afin d'analyser les parcours des individus selon les caractéristiques concernées. Concrètement, l'analyse se fonde sur l'établissement de tables de mortalité à extinctions multiples en calculant les probabilités conditionnelles d'occurrence et celles de vivre une transition donnée entre deux évènements. Ainsi, le nombre de femmes vivant une transition est rapporté au nombre total de femmes exposées au risque de la vivre (celles n'ayant pas encore vécu ladite transition et qui sont toujours

3. L'existence inévitable d'un intervalle ouvert à droite (vers le futur), biais majeur de toute collecte de données rétrospectives (Courgeau et Lelièvre, 1989), nous oblige à prendre en compte la censure et les troncatures à droite. En somme, les femmes n'ayant pas encore terminé leur période reproductive sont encore soumises au risque d'avoir un enfant une fois l'enquête réalisée. L'impact majeur de la censure à droite se retrouve pour les cohortes enquêtées les plus jeunes. Nos résultats ont été interprétés en tenant compte de cet aspect important présent dans les données biographiques de l'enquête FFS. 
en observation). Selon Rajulton, plusieurs types de transitions sont possibles à partir d'un même état initial; celles-ci sont abordées comme des risques concurrents (1999). Ici, il est question de la probabilité finale qu'une femme ait d'expérimenté complètement l'une ou l'autre des trajectoires avant la première maternité (probabilité de trajectoire ou d'itinéraire) afin d'identifier les différentes intensités des principales trajectoires vécues par les femmes dans les trois régimes.

La perspective «state space approach» et le format utilisé par le programme «Lifehist» appliqués à des données réelles se basent sur l'âge des individus au moment où ils vivent les différents évènements concernés. Par le calcul du mois et de l'année de l'évènement par rapport au mois et l'année de naissance de la femme, nous pouvions, au mois près, avoir l'âge des femmes au moment de l'occurrence de chaque évènement. Les questions permettant de déterminer l'âge des femmes au moment de l'occurrence des évènements ont toutes été posées de manière à interroger par rapport à la date de l'évènement et non par rapport à l'âge des femmes. Ce choix limite les erreurs typiques des collectes de données rétrospectives où les omissions ou les oublis chez une femme concernant l'âge qu'elle avait lorsqu'un événement est survenu plusieurs années auparavant (Courgeau et Lelièvre, 1989).

Dans une analyse de type causal, la variable se référant à l'âge de la mère au moment de la naissance du "premier enfant» serait appelée la variable dépendante. Selon Rajulton et dans le cas de «state space approach », il est question d'un événement absorbant. Il est, par son caractère définitif, l'événement final étudié dans les trajectoires; autrement dit, les séquences d'évènements étudiées se terminent au moment où l'événement absorbant se produit (la venue du premier enfant, moment auquel nous enregistrons l'âge de la mère). Il représente pour notre analyse l'arrêt de l'observation des trajectoires indifféremment du nombre d'évènements vécus auparavant ou après. Les évènements gravitant autour et menant à la première maternité sont de type transient (Rajulton, 1999): ils permettent aux individus le passage d'un état à un autre (par exemple le mariage fait passer de l'état de célibataire à l'état de marié). À la différence des évènements absorbants, les évènements transients permettent autant de sortir d'un état que d'y entrer. Ce sont ces évènements et leur enchaînement qui tracent le parcours vers la première maternité dans les échantillons étudiés.

Une dernière considération concernant ces événements est qu'ils sont de type non récurrent, ils ne peuvent se produire qu'une seule fois, il s'agit de l'âge des femmes à la première mise en union, à la deuxième union, à la fin des études, au premier emploi, etc. Un certain nombre d'évènements transients de type familial/conjugal ont été rassemblés afin d'identifier les cheminements possibles par rapport à la littérature existante sur le passage à la vie adulte:

- «L'indépendance» des femmes face à leur famille d'origine ${ }^{4}$;

4. Question posée: «À quel mois et quelle année avez-vous quitté pour la première fois vos parents pour vivre par vos propres moyens?» En Italie, il s'agit de l'indépendance financière, alors qu'en Suède et en France, on parle d'indépendance résidentielle. 
- La mise en union ${ }^{5}$ : la première mise en union en cohabitation hors mariage, la première mise en union dans le cadre du mariage, la deuxième mise en union en cohabitation hors mariage, la deuxième mise en union dans le cadre du mariage, etc.

Pour des raisons techniques (soit le nombre maximal d'évènements pouvant être intégrés dans le programme) et d'interprétation, nous avons séparé ces évènements de ceux de type scolaire/professionnel tout en y conservant l'évènement de l'indépendance de la famille d'origine qui est importante dans les deux types de trajectoires:

- La fin de la formation académique principale;

- Le premier emploí.

\section{RÉSULTATS}

\section{Les Italiennes, homogénéité des parcours de vie et importance de l'institution du mariage}

Le régime familialiste italien présente une mobilisation des ressources de type familial/ conjugal assez homogène. En effet, durant les décennies observées, la répartition des Italiennes au sein des différentes trajectoires montre qu'elles empruntent largement un parcours unique avant d'avoir un premier enfant avec une présence majoritaire dans la trajectoire 5 avec 67,5\% (tableau 1).

La particularité de celle-ci est qu'elle se fonde sur le mariage comme condition obligée de création de la famille, il est d'ailleurs le seul événement vécu. Rappelons que dans le cas de la collecte italienne, le mariage direct permet, d'une part, le passage du statut de célibataire (sous la dépendance familiale) au statut de femme mariée («indépendante» de la famille d'origine) et, d'autre part, permet aux femmes de se déclarer indépendantes financièrement. En outre, cette trajectoire illustre le comportement traditionnel des Italiennes, par un fort attachement à la répartition traditionnelle des rôles et la simultanéité d'un certain nombre de changements déterminants: indépendance envers la famille d'origine, fin du célibat et mariage. S'il y a lieu, l'expérimentation de la vie de couple en fréquentant son partenaire hors du contexte de la famille et, en pouvant évaluer si le partenaire répond aux attentes et aux désirs avant l'engagement, s'effectue ainsi «sous l'œil» de la famille d'origine. De fait, le choix est décisif

5. Les partenaires successifs et le type d'union vécu. La question d'origine pour la cohabitation hors mariage était: "À quel mois et quelle année avez-vous pour la première fois commencé à vivre dans le même ménage avec votre (premier, deuxième) partenaire?» Pour le mariage (avec le premier partenaire, le deuxième, etc.), les trois pays ont recensé la date de l'évènement, peu importe si la cohabitation avec le mari fut simultanée ou non.

6. Toutes les séquences d'emploi étaient concernées par la collecte, grâce à la date de début et la date de fin. La question suivante était posée: "À quel mois et quelle année avez-vous commencé votre (premier, deuxième, etc.) emploi?» Notons que si en Italie et en Suède les données sont relativement complètes (elles rapportent les différentes séquences d'emploi successives), en France, la comparabilité des données pose certains problèmes ( $1^{\text {er }}$ emploi seulement). Pour cela, nous utilisons seulement la première séquence d'emploi pour chacun des pays, déterminante de tout évidence du passage à l'âge adulte (Galland). 
Tableau 1: Trajectoires familiale/conjugale et scolaire/professionnelle avant la première maternité des ITALIENNES, selon la cohorte

\begin{tabular}{|c|c|c|c|c|c|c|c|}
\hline $\begin{array}{l}\text { Détails trajectoires familiale/ } \\
\text { conjugale }\end{array}$ & TOTAL & Plus 35 ans & Moins 35 ans & Détails trajectoires scolaire/professionnelle & TOTAL & Plus 35 ans & Moins 35 ans \\
\hline Vivre chez les parents* & 5,3 & 3,4 & 11,6 & $\left(\mathrm{TRP}_{1}\right)$ Ind-ENFT & 3,5 & 6,1 & 1,3 \\
\hline (TRF1) Ind-M-ENFT & 8,7 & 8,5 & 8,2 & (TRP2) Ind-empl-ENFT & 0,5 & 0,9 & 0,2 \\
\hline (TRF2) Ind-C-ENFT & 0,7 & 0,5 & 0,8 & (TRP 3 ) Ind-Empl-Études-ENFT & 1,2 & 1,6 & 0,8 \\
\hline$\left(\mathrm{TRF}_{3}\right)$ Ind-C-M-ENFT & 1,4 & 1,0 & 2,0 & (TRP4) Ind-Études-Empl-ENFT & 1,9 & 1,8 & 1,7 \\
\hline$\left(\mathrm{TRF}_{4}\right)$ Ind-C1-C2-ENFT & $\circ$ & ० & ० & (TRP5) Études-Ind-ENFT & 27,0 & 28,3 & 25,4 \\
\hline (TRF5) Ind/M-ENFT & 67,5 & 72,7 & 61,5 & (TRP6) Études-Ind-Empl-ENFT & 4,1 & 4,1 & 3,6 \\
\hline (TRF6) Ind/C-ENFT & 1,4 & 0,8 & 2,2 & (TRP7) Études-Empl-Ind-ENFT & 34,3 & 30,3 & 38,5 \\
\hline (TRF7) Ind/C-M-ENFT & 2,8 & 1,5 & 4,4 & (TRP8) Empl-Ind-ENFT & 2,2 & 3,6 & 1,0 \\
\hline (TRF8) Ind/C1-C2-ENFT & $\circ$ & 0,1 & ० & (TRPg) Empl-Ind-Études-ENFT & 1,5 & 1,8 & 1,8 \\
\hline (TRF9) ENFT & 2,4 & 2,8 & 2,6 & (TRP10) Empl-Études-Ind-ENFT & 7,1 & 7,3 & 9,6 \\
\hline TOTAL & 84,9 & 87,9 & 81,7 & TOTAL & 83,3 & 85,8 & 83,9 \\
\hline
\end{tabular}

Probabilités conditionnelles des trajectoires (\%)

* Ces femmes n'ont pas encore quitté leurs parents 
puisque le mariage, fortement institutionnalisé, scelle définitivement l'union et bâtit les fondations de la future famille. Ainsi, l'exclusivité attribuée au premier partenaire, par le biais du mariage direct, est une particularité essentielle du régime italien dans le parcours vers la première maternité où l'institution du mariage conserve le statut privilégié et quasi unique de fondateur de la famille (Pinelli et al., 2001).

D'autres formes de cheminements observées vers la première maternité des Italiennes montrent que certaines ont obtenu leur indépendance «financière» sans pour autant se mettre en couple. Elles sont toutefois peu nombreuses, avec 8,7 \% (traj. 1, Ind-M-Enft). Si, en Italie, expérimenter une phase d'indépendance financière n'est pas pour autant un prélude à une diversité de combinaisons et un enchaînement de plusieurs séquences, les femmes ayant vécu cette trajectoire ont toutefois un comportement différent de celles de la précédente trajectoire. La préalable indépendance financière les sort du cadre exclusivement traditionnel en expérimentant une certaine autonomie par rapport à leur famille d'origine tout en étant affranchies en partie ou en totalité de leur futur mari, qui n'est plus le seul garant de l'apport des ressources nécessaires pour faire vivre la famille. Il s'agit là d'une variante de la répartition traditionnelle des rôles, où l'homme est pourvoyeur des besoins de la famille (Njoki, 2006).

Les deux trajectoires précédentes regroupent 76,2\% de l'échantillon italien et révèlent l'importance que les femmes ont attribuée au fait d'être mariée avant d'avoir un enfant. La faible présence des Italiennes dans les sept autres trajectoires démontre le faible attrait pour la cohabitation et les naissances hors mariage durant la période observée, peu importe que la cohabitation représente l'encadrement privilégié de la maternité ou une étape test avant le mariage (Cicchelli et al., 2007). Ainsi, seulement 2,1 \% des Italiennes de l'échantillon ont vécu une première naissance et une cohabitation hors mariage tout en excluant le mariage dans leur trajectoire (voir traj. 2, 4, 6 et 8). Le mariage était un évènement essentiel dans la vie des Italiennes en jouant le rôle de fondement nécessaire à la création de la famille.

Cette forte homogénéité des trajectoires familiales/conjugales des Italiennes a persisté à travers le temps et a contribué à renforcer la position particulière du régime familialiste et traditionaliste italien de la fin des années 1990 (Cassese, 2001). En effet, le modèle principal de cheminement vers la première maternité (traj. 5) a conservé son importance relative durant les décennies observées, avec 72,7 \% de probabilités de vivre un mariage direct suivi d'une première naissance pour les plus âgées contre $61,5 \%$ pour les plus jeunes. Ce léger recul des plus jeunes Italiennes ne reflète pas pour autant l'expérimentation de trajectoires alternatives (comprenant par exemple la cohabitation hors mariage), elles restent au contraire chez leurs parents (11,6\%). De fait, elles ne modifiaient pas leurs comportements en fonction des nouveaux comportements familiaux en expansion dans les pays occidentaux, elles choisissaient plutôt de décrocher du modèle traditionnel, d'une façon inattendue, en optant pour une stratégie d'attente et en retardant leur engagement au sein de la «vie adulte». C'est une hypothèse fortement possible, compte tenu des difficultés qu'a éprouvées et qu'éprouve toujours le régime familialiste italien, n'étant pas en mesure d'ajuster les 
dynamiques socio-économiques aux modèles qui se pratiquent ailleurs (comme l'important problème de logement et le chômage élevé des jeunes (Cicchelli et al., 2007).

\section{De l'homme pourvoyeur à la contribution des femmes au revenu du ménage}

La construction des trajectoires de type scolaire/professionnel des Italiennes illustre également une mobilisation des ressources très traditionnelle durant les décennies observées, malgré une certaine variété de trajectoires expérimentées.

La trajectoire privilégiée par les Italiennes enchaîne «la fin des études», «le premier emploi » puis «l'indépendance familiale», préalablement à la naissance du premier enfant, avec 34,3 \% de l'échantillon (traj. 7). Ce parcours enchaîne les évènements clés à l'image du parcours traditionnel du passage à l'âge adulte (Galland, 2000) avec une logique «sécuritaire». La fin des études procure le premier changement de statut significatif dans la vie des femmes, puis elles sont prêtes à accéder au marché du travail, garant d'une situation financière plus favorable. Cette trajectoire montre également une importante séparation entre les évènements de type scolaire/professionnel (premier évènement «fin des études», deuxième évènement "premier emploi ») et les évènements familiaux/conjugaux, car c'est «l'indépendance» qui marque la prochaine transition, ce qui signifiait pour bon nombre d'Italiennes la mise en couple simultanée à l'indépendance. Pourtant, si certaines Italiennes ont obtenu leur indépendance financière en grande partie grâce au mariage, d'autres avaient déjà travaillé avant de se marier, et ce, même si elles ne se déclaraient pas indépendantes financièrement par leur emploi. La fin des études et les débuts sur le marché du travail se sont effectués pour ces dernières dans le cadre familial (du moins d'un point de vue financier), puis la mise en couple a accordé la pleine indépendance et la garantie suffisante pour la fondation d'une famille. Le découpage séquentiel des dimensions de la vie de ces femmes laisse supposer qu'elles ont eu de la difficulté à retourner sur le marché du travail après la maternité (accentuée d'ailleurs par le manque de systèmes de garde) (Croisetière, 2006) ou du moins que le retour au travail des femmes s'est effectué lorsque la dimension familiale pouvait être mise à nouveau de côté (une fois les enfants plus grands) (Cooke, 2006). Cette trajectoire sécuritaire a même été privilégiée par les plus jeunes lors des décennies observées avec 38,5\% contre 30,3\% pour les plus âgées.

Certaines Italiennes présentaient, par ailleurs, un traditionalisme encore plus marqué en n'ayant eu aucune séquence d'emploi avant de vivre une première maternité (traj. 5: 27,0\%). Elles ont toutefois terminé leurs études avant de se déclarer indépendantes financièrement et d'avoir un premier enfant. Cette trajectoire traditionnelle à l'état pur (Sauvain-Dugerdil, 2005b) indique une adhésion à une logique familialiste où la répartition des rôles de genre dans la famille est très marquée. Il n'existe pas chez ces Italiennes de séparation stratégique entre la dimension famille et la dimension professionnelle, puisqu'elles se sont consacrées exclusivement à la première. Toutefois, certaines Italiennes ont cumulé plusieurs statuts avant d'accéder à leur première maternité, comme celles dont l'enchaînement présente une séquence 
d'emploi tout en étant encore aux études, puis qui ont mis l'accent par la suite sur la dimension conjugale pour fonder une famille (traj. 10: 7,1\%).

L'observation à travers le temps de la mobilisation des ressources de type scolaire/ professionnel vers la première maternité a montré que les plus jeunes étaient plus nombreuses à participer au marché du travail: 57,2\% vivent une trajectoire menant à un premier enfant avec au moins une séquence d'emploi, contre $51,4 \%$ pour les plus âgées (somme des traj. 1, 2, 3, 5, 6, 7, 8, 9 et 10). De fait, l'expérience des trajectoires ne présentant aucune séquence d'emploi a diminué durant les décennies observées (traj. 1 de $6,1 \%$ à $1,3 \%$; traj. 5 de $28,3 \%$ à 25,4\%). Il n'en demeure pas moins que la stratégie séquentielle des Italiennes, forte d'aspects traditionnels, s'est perpétuée à travers le temps puisque les plus jeunes ont expérimenté de même que leurs aînées l'indépendance financière au moment du mariage (traj. 7 et 10). Cette stratégie se distingue nettement des trajectoires de repli observées dans le cheminement familial/ conjugal.

\section{Les Suédoises, diversité des parcours de vie teintés de stratégies de conciliation}

La construction des trajectoires de type familial/conjugal au sein de l'échantillon des Suédoises fait ressortir une tendance intéressante; elles se sont éparpillées dans plusieurs trajectoires présentant une variété de combinaison des évènements avant la naissance de leur premier enfant. Cette hétérogénéité présente toutefois une concentration plus importante pour les trajectoires 2, 3 et 6 . Étant assez différente l'une de l'autre, la diversité des choix familiaux et conjugaux des Suédoises était d'autant plus grande.

Une forte concentration des Suédoises de notre échantillon, soit 22,9 \% (tableau 2), a choisi de fonder une famille et d'avoir des enfants dans le cadre d'une cohabitation hors mariage. Ces Suédoises ont expérimenté une période de célibat, indépendamment de la cellule familiale d'origine, se sont mises ensuite en couple hors mariage et ont eu un enfant dans le cadre de cette union (traj. 2, Ind-Cohab-Enft). L'autonomie relative de ces Suédoises ne provenait dès lors ni des parents ni du partenaire, mais probablement d'un apport personnel de ressources, quoiqu'une contribution des parents ne puisse être exclue; elle vient cependant s'ajouter à une forme d'autonomie qui se concrétise dans la formation d'un ménage indépendant. Ces femmes ont ainsi tourné le dos au mariage comme élément fondateur de la famille encouragé par un cadre culturel et institutionnel adéquat pour vivre une cohabitation hors mariage féconde.

Si certaines Suédoises ont considéré le mariage comme un élément important et déterminant pour fonder leur famille, c'est la place attribuée à celui-ci qui n'était pas de la même nature que ce que nous avons pu voir chez les Italiennes. En effet, elles préféraient encadrer la naissance de leur premier enfant dans le mariage en ayant préalablement vécu une période de vie commune hors mariage avec leur conjoint (traj. 3, Ind-Cohab-Mar-Enft: 14,9\%). Ce cheminement par étapes vers le mariage et la fondation de la famille peut être interprété de plusieurs manières: il permet par la 


\begin{tabular}{|c|c|c|c|c|c|c|c|}
\hline $\begin{array}{l}\text { Détails trajectoires familiale/ } \\
\text { conjugale }\end{array}$ & TOTAL & Plus 35 ans & Moins 35 ans & Détails trajectoires scolaire/professionnelle & TOTAL & Plus 35 ans & Moins 35 ans \\
\hline Vivre chez les parents** & 0,1 & 0,1 & o & $(\mathrm{TRP} 1)$ Ind-ENFT & 5,4 & 7,3 & 4,2 \\
\hline (TRF1) Ind-M-ENFT & 4,5 & 5,8 & 3,8 & (TRP2) Ind-empl-ENFT & 10,9 & 15,9 & 7,7 \\
\hline (TRF2) Ind-C-ENFT & 22,9 & 21,0 & 24,1 & (TRP3) Ind-Empl-Études-ENFT & 12,9 & 10,7 & 12,4 \\
\hline$\left(\mathrm{TRF}_{3}\right)$ Ind-C-M-ENFT & 14,9 & 18,8 & 11,4 & (TRP4) Ind-Études-Empl-ENFT & 10,9 & 7,2 & 13,3 \\
\hline (TRF4) Ind-C $1-C_{2}-E N F T$ & 5,0 & 2,4 & 7,3 & (TRP5) Études-Ind-ENFT & 0,2 & 0,2 & o \\
\hline (TRF5) Ind/M-ENFT & 4,6 & 6,4 & 3,0 & (TRP6) Études-Ind-Empl-ENFT & 2,6 & 1,8 & 2,8 \\
\hline (TRF6) Ind/C-ENFT & 13,8 & 12,0 & 15,0 & (TRP7) Études-Empl-Ind-ENFT & 11,6 & 7,2 & 14,8 \\
\hline (TRF7) Ind/C-M-ENFT & 7,3 & 10,2 & 5,1 & (TRP8) Empl-Ind-ENFT & 14,5 & 24,3 & 8,0 \\
\hline (TRF8) Ind/C1-C2-ENFT & 4,5 & 2,2 & 6,1 & (TRPg) Empl-Ind-Études-ENFT & 15,8 & 10,7 & 20,3 \\
\hline (TRF9) ENFT & 2,4 & 4,7 & 1,0 & (TRP1o) Empl-Études-Ind-ENFT & 3,7 & 3,1 & 4,0 \\
\hline TOTAL & 79,9 & 83,5 & 76,8 & TOTAL & 88,5 & 88,4 & 87,5 \\
\hline
\end{tabular}

Probabilités conditionnelles de trajectoires (\%)

* Ces femmes n'ont pas encore quitté leurs parents 
vie commune hors mariage, d'expérimenter la relation de couple et d'évaluer si elle répond aux attentes, puis, par une première indépendance de vie en célibataire, de vivre une période d'autonomie hors du contexte de la famille d'origine et de la famille qu'elles fondent elles-mêmes. Les Suédoises pouvaient vivre différents statuts avant de s'engager dans la maternité.

En outre, certaines Suédoises se sont engagées plus rapidement dans leur processus de création de la famille tout en rejetant l'institution du mariage: celles-ci ont choisi l'union directe de type cohabitation hors mariage (traj. 6, Ind/Cohab-Enft: $13,8 \%$ ). Moins traditionnelles face à la famille, elles ont toutefois eu en commun avec le traditionnel mariage direct d'expérimenter leur première indépendance à travers une mise en union, c'est-à-dire de transposer leur dépendance envers leur famille d'origine en une interdépendance avec le conjoint (Brachet, 2001).

Si les Suédoises se sont concentrées principalement dans les trois trajectoires maternelles ci-dessus, elles ont expérimenté l'ensemble des neuf trajectoires principales avec 28,3\% de l'échantillon pour les six autres trajectoires. Sans en présenter le détail, cette diversité des «carrières maternelles " gagne à être relevée par quelques caractéristiques. En effet, avec une préférence pour la cohabitation hors mariage (traj. 2, 4, 6 et 8:46,2\%) au détriment du mariage (traj. 1, 3, 5 et 7:31,3\%), les Suédoises sont incontestablement les précurseuses des nouveaux comportements familiaux observés au cours des dernières décennies (Pauti, 1992). La trajectoire privilégiée des Italiennes a été faiblement suivie en Suède (traj. 5: 4,6 \%). En outre, les Suédoises qui se sont mariées avant d'avoir un premier enfant sont plus nombreuses à avoir vécu avant avec leur partenaire le mariage en cohabitation. Notons par ailleurs que la phase d'expérimentation de la vie en couple et/ou d'indépendance de la vie familiale d'origine avant d'avoir un premier enfant a augmenté grandement le nombre d'évènements vécus par les Suédoises avant leur première maternité (59,1 \% ont expérimenté au moins deux évènements, somme des traj. 1, 2, 3, 4, 7 et 8). Le choix de vivre ou non de manière indépendante de la famille d'origine avant de se mettre en union marque également cette diversité. Certaines ont choisi de quitter leurs parents pour vivre seules et célibataires (traj. 1, 2, 3 et 4:47,3\%), d'autres, au contraire, ont marqué le départ de chez leurs parents par une mise en union directe (traj. 5, 6,7 et 8:30,2\%).

Tout au long des décennies observées, la diversité familiale/conjugale suédoise a été constante tout en se modifiant, voire en s'accentuant. Essentiellement, il s'agit de la nature de l'union pour encadrer la première maternité qui s'est modifiée; il y a une véritable inversion pour ce qui est des naissances dans le cadre du mariage (avec $41,2 \%$ pour les plus âgées et $23,3 \%$ pour les plus jeunes) et les naissances hors mariage (avec 37,6 \% pour les plus âgées et 52,5\% pour les plus jeunes) (Pinelli et al., 2001). Compte tenu des développements en matière de modèles familiaux, la préférence pour la cohabitation hors mariage peut à long terme accentuer cette diversité et conduire à la multiplication de différents partenaires de vie, car il est plus facile de rompre les unions et d'en expérimenter plusieurs lorsqu'elles ne sont pas scellées par le mariage (Munoz-Perez, 1986). En somme, l'éclatement des trajectoires dans le 
modèle social-démocrate suédois, à travers le temps, a nettement remis en question l'existence d'un cadre majoritaire pour la création de la famille en renforçant la liberté d'action dans les parcours de vie.

\section{Stratégies de conciliation et parcours travail/famille réconciliés}

La construction des trajectoires de type scolaire/professionnel des Suédoises montre que, tout au long des décennies observées, la mobilisation de leurs ressources se partage entre leur carrière professionnelle et maternelle. Le cumul de différents statuts en est une manifestation: travailleuse/étudiante, mère/étudiante, à la recherche du partenaire idéal tout en lançant sa carrière, etc.

Ainsi, une des trajectoires privilégiées par les Suédoises démarre par une séquence d'emploi tout en vivant chez les parents et tout en étant aux études (traj. 9, Empl-IndÉtudes-Enft avec 15,8\%; tableau. 2). Ces jeunes femmes recherchent une certaine autonomie (du moins financière) dans leur vie, puisqu'elles n'hésitaient pas à cumuler plusieurs statuts en même temps pour l'obtenir. Cette trajectoire montre également un besoin hâtif de se responsabiliser puisque le deuxième évènement marquant est de quitter les parents. Si cela n'indique pas forcément un cumul du statut d'étudiante et du travail, cette trajectoire présente toutefois une séquence d'indépendance résidentielle où les femmes, tout en vivant hors du contexte familial, sont aux études et travaillent en même temps. Cette situation était déjà largement appuyée par le régime suédois puisque les aides envers les jeunes sont nombreuses depuis les années 1970 (Mc Donald, 1997) — notamment la gratuité des études postsecondaires. L'examen de cette trajectoire conduit à remettre en question l'idée que les femmes, qui commencent leur vie d'adulte par l'emploi, auront des carrières plus accaparantes et mettront moins l'accent sur la fondation d'une famille — Galland (2000 et 2005) les appelait les carriéristes - ; elles peuvent cumuler au contraire plusieurs statuts en même temps et présentent de fortes stratégies de conciliation et une liberté d'action, caractéristiques peu étonnantes de ce régime (Mathieu, 2003).

Ces stratégies de conciliation et le cumul des statuts étaient encore plus marqués pour celles qui n'avaient pas terminé leurs études au moment de la naissance de leur premier enfant (traj. 8, Empl-Ind-Enft avec 14,5\%). Expérimenter les doubles statuts travail/études et études/maternité (hors contexte de la famille d'origine) est en Suède tout à fait réalisable grâce aux fortes politiques défamilialisantes, réel soutien aux mères-étudiantes (par les services de garde, les aides financières, etc.) et aux couples post-maternité (McDonald, 2002).

Les avantages du régime suédois ont permis également à certaines de vivre les différentes étapes du "passage à la vie d'adulte», comme l'emploi et la fin des études, hors du contexte familial (voir traj. 2 avec 10,9 \%: Ind-Empl-Enft; traj. 3 avec 12,1 \%: Ind-Empl-Études-Enft et traj. 4 avec 10,9\%: Ind-Études-Empl-Enft). Les Suédoises pour lesquelles le fait de «quitter les parents» est la première séquence de leur trajectoire présentent une autonomie d'autant plus grande qu'elles ont accès à des stratégies conciliantes par le cumul de statuts différents. 
Enfin, certaines Suédoises ont choisi d'emprunter des parcours un peu plus sécuritaires que les précédentes avec des profils travail/famille moins conciliants (traj. 7 , Études-Empl-Ind-Enft avec 11,6 \%). Ici, les évènements s'enchaînent conformément à la trajectoire traditionnelle du passage à l'âge adulte (Galland, 2005).

Si tout au long des décennies observées, la diversité des trajectoires scolaire/professionnelle et les stratégies de conciliation avec la possibilité de cumuler plusieurs statuts figurent le modèle suédois, ces phénomènes se sont d'autant plus amplifiés que le nombre de séquences scolaire/professionnelle chez les plus jeunes ont augmenté. Ainsi, la variété des trajectoires alternatives permettant une relative liberté de conciliation chez les plus âgées (traj. 8 avec $24,3 \%$ et traj. 2 avec 15,9\%) s'est nettement accentuée chez les plus jeunes qui se sont éparpillées dans des trajectoires cumulant plusieurs statuts (traj. 9 avec $20,3 \%$; traj. 7 avec $14,8 \%$ ).

\section{Les Françaises, diversité des parcours de vie teintés d'un certain traditionalisme}

Le régime conservateur français présente des cheminements de type familial/conjugal, à mi-chemin entre le rassemblement traditionnel à l'italienne et l'éparpillement à la suédoise illustrant la liberté des femmes de choisir l'itinéraire idéal.

La voie privilégiée italienne d'accession à la première maternité est, bien que moins présente, tout aussi importante dans l'échantillon français avec 27,7 \% (traj. 5 : tableau 3). Rappelons qu'avec le mariage direct comme seul événement préalable à la première maternité, cette trajectoire dans le passage d'un statut à l'autre attribue une position privilégiée au mari. S'il y avait en France un certain traditionalisme à l'italienne à travers l'importance de cette trajectoire, il est de rigueur de rappeler qu'il n'était pas de la même nature dans la mesure où la simultanéité ne signifiait pas une indépendance économique mais plutôt résidentielle. Le départ de chez les parents pour une mise en couple directe illustre tout de même la sécurité que recherchent les Françaises dans leur vie, puisqu'elles ne vivent pas seules en logement. En outre, les jeunes Françaises ont exprimé leur rejet de ce modèle traditionnel avec 12,8 \% contre $42,4 \%$ pour les plus âgées (traj. 5).

D'autres ont plutôt favorisé un processus vers la création de la famille hors du cadre du mariage. Elles ont quitté le foyer parental pour se mettre en union directe par une cohabitation féconde (traj. 6 avec 13,5\%). Si le mariage n'est donc pas toujours indispensable pour légitimer une union; une sorte d'exclusivité est toutefois attribuée au premier partenaire car elles n’ont pas expérimenté d'autres séquences avant de fonder une famille (Malpas, 1999).

La cohabitation hors mariage après une période de célibat hors du foyer parental (traj. 2) a été vécue par 10,7 \% de l'échantillon des Françaises. Celles-ci fondent une famille sans accorder une haute importance à la légitimation par le mariage de leur union et de leur maternité. En outre, la période de célibat hors de leur famille d'origine révèle le développement d'une certaine autonomie avant de trouver le partenaire de vie idéal puisque celle-ci facilite la fréquentation informelle des jeunes couples avant de franchir le pas de s'installer ensemble. D’autres, après avoir vécu seules, puis en 
Tableau 3: Trajectoires familiale/conjugale et scolaire/professionnelle avant la première maternité des FRANÇAISES, selon la cohorte

\begin{tabular}{|c|c|c|c|c|c|c|c|}
\hline $\begin{array}{l}\text { Détails trajectoires familiale/ } \\
\text { conjugale }\end{array}$ & TOTAL & Plus 35 ans & Moins 35 ans & Détails trajectoires scolaire/professionnelle & TOTAL & Plus 35 ans & Moins 35 ans \\
\hline Vivre chez les parents** & 0,9 & 1,1 & 1,4 & $\left(T_{R P}\right)$ Ind-ENFT & 4,3 & 6,0 & 2,7 \\
\hline (TRF1) Ind-M-ENFT & 7,1 & 11,0 & 2,2 & (TRP2) Ind-empl-ENFT & 1,9 & 2,6 & 1,1 \\
\hline (TRF2) Ind-C-ENFT & 10,7 & 7,5 & 15,0 & (TRP3) Ind-Empl-Études-ENFT & 3,7 & 3,5 & 4,0 \\
\hline (TRF3) Ind-C-M-ENFT & 10,6 & 10,6 & 11 & (TRP4) Ind-Études-Empl-ENFT & 7,7 & 6,0 & 9,8 \\
\hline (TRF4) Ind-C1-C2-ENFT & 1,8 & 1,0 & 2,7 & (TRP5) Études-Ind-ENFT & 8,6 & 6,6 & 9,5 \\
\hline (TRF5) Ind/M-ENFT & 27,7 & 42,4 & 12,8 & (TRP6) Études-Ind-Empl-ENFT & 12,6 & 8,4 & 17,5 \\
\hline (TRF6) Ind/C-ENFT & 13,5 & 4,8 & 22,4 & (TRP7) Études-Empl-Ind-ENFT & 34,6 & 34,6 & 34,7 \\
\hline (TRF7) Ind/C-M-ENFT & 8,1 & 5,7 & 10,5 & (TRP8) Empl-Ind-ENFT & 8,5 & 14,0 & 3,3 \\
\hline (TRF8) Ind/C1-C2-ENFT & 1,4 & 0,2 & 2,8 & (TRPg) Empl-Ind-Études-ENFT & 1,6 & 1,8 & 1,3 \\
\hline (TRF9) ENFT & 4,3 & 3,9 & 4,2 & (TRP10) Empl-Études-Ind-ENFT & 5,3 & 5,6 & 4,9 \\
\hline TOTAL & 85,2 & 87,1 & 83,6 & TOTAL & 88,8 & 89,1 & 88,8 \\
\hline
\end{tabular}

Probabilités conditionnelles de trajectoires (\%)

* Ces femmes n'ont pas encore quitté leurs parents 
cohabitation, ont choisi de légitimer leur union par le mariage avant d'enfanter (traj. 3 avec 10,6 \%). Il s'agit somme toute d'un cheminement vers le premier enfant assez sécuritaire, où les femmes ont testé différents statuts avant de s'engager dans la maternité.

Il est intéressant de constater de façon globale que les Françaises ont encadré majoritairement la venue de leur premier enfant par le mariage (traj. 1, 3, 5 et 7 avec $53,5 \%)$ et ont usé de la mise en couple directe comme démarrage de leur trajectoire (traj. 5, 6, 7 et 8 avec 50,7 \%). Contrairement aux Italiennes, le mariage comme légitimation a été plus fréquemment précédé d'une cohabitation hors mariage avec 18,7\% (traj. 3 et 7) ou d'une période d'indépendance hors de la famille d'origine avec 7,1\% (traj. 1). L'attachement au mariage y était différent puisqu'elles ont accumulé différents statuts avant celui-ci, ce qui augmente d'autant plus le nombre de séquences vécues (avec une: 41,2\%; deux: 27,3\%; trois: 12,4\%). Enfin, rares sont celles qui ont eu leur premier enfant avec un deuxième partenaire avec seulement 3,2 \% (traj. 4 et 8 ).

Si le modèle français de mobilisation des ressources familiales/conjugales présente un attachement relativement fort aux valeurs traditionnelles, l'analyse à travers le temps montre que les Françaises ont expérimenté des trajectoires qui ressemblent très fortement au profil traditionnel, alors que les plus jeunes s'identifient largement au profil comprenant des trajectoires alternatives à l'instar des nouveaux comportements familiaux (Galland, 2000).

La préférence des jeunes Françaises n’était pas tournée vers la vie de célibataire hors du cadre familial (comme c'était le cas en Suède) mais davantage vers la cohabitation directe hors mariage (traj. 6: 22,4 \%; traj. 7: 10,5\%) au lieu du mariage direct. Ces deux trajectoires ne représentent que $4,8 \%$ et $5,7 \%$ respectivement pour les plus âgées. La particularité des plus jeunes est donc d'avoir rejeté le mariage (avec 36,5\% pour les plus jeunes et $69,7 \%$ pour les plus âgées), privilégiant davantage la cohabitation comme encadrement des naissances, avec $42,9 \%$ pour les plus jeunes et $13,5 \%$ pour les plus âgées. Ainsi, l'expérience du célibat hors du contexte familial n'est pas un choix privilégié par les plus jeunes Françaises, à l'instar des Suédoises. Certaines nuances sont nécessaires puisqu'elles ont conservé un certain traditionalisme dans l'engagement en couple à travers l'exclusivité accordée au conjoint.

\section{Entre des parcours séquentiels et le rejet du traditionalisme}

L'observation des trajectoires de type scolaire/professionnel des Françaises montre qu'elles ont expérimenté un profil mixte, à mi-chemin entre le traditionalisme des Italiennes et le cumul de statuts des Suédoises. Véritable conciliation à stratégie davantage séquentielle, les probabilités de n'avoir jamais travaillé sont nuancées et la formation scolaire terminée demeure une condition d'accès à la maternité (Nicole-Drancourt, 2006).

Durant les décennies observées, elles ont vécu à 34,6 \% la trajectoire traditionnelle, privilégiant ainsi une séquence d'évènements offrant une certaine sécurité à travers le cadre familial d'origine (traj. 7, Études-Empl-Ind-Enft). Deux nuances sont 
à apporter à cette trajectoire: premièrement, «quitter les parents » est, dans ce contexte, soit un changement de résidence pouvant être vécu simultanément avec une mise en union de type cohabitation hors mariage, soit être le commencement d'une période de célibat avant de se mettre en couple; deuxièmement, «le premier emploi» n'est pas forcément l'emploi idéal recherché et des emplois subséquents ont pu être occupés avant la première maternité. Il n'en demeure pas moins que cette trajectoire illustre un parcours sécuritaire où la conciliation séquentielle octroyant un poids à la carrière puis à la dimension familiale présente une certaine recherche de stabilisation avant de fonder une famille et représente la trajectoire traditionnelle type (Cavalli et al., 1993). Mais ce qui est intéressant de constater, c'est la persistance à travers le temps des Françaises au sein de cette trajectoire. Celle-ci figure la persistance d'un modèle présentant un aspect séquentiel: les dimensions familiale/conjugale et scolaire/professionnelle y sont bien séparées (Sauvain-Dugerdil, 2005b). En outre, ces femmes n'ont pas cumulé les statuts d'étudiante-travailleuse ou d'étudiante-mère (traj. 5 dans tableau. 3 ). Cette même logique sécuritaire se retrouve d'une certaine manière chez celles qui ont terminé leur formation dans le cadre de la famille d'origine, ont quitté les parents puis ont commencé leur premier emploi (traj. 6 avec 12,6\%). Il s'agit pour celles-ci d'une stratégie sécuritaire par la séparation des dimensions du parcours de vie où la mise en union prend une part importante dans la fondation de sa propre famille. L'expérience de cette trajectoire est d'autant plus intéressante au modèle conservateur français, qu'elle prend de l'ampleur à travers le temps avec 17,5\% pour les plus jeunes contre $8,4 \%$ pour les plus âgées.

Cette logique sécuritaire s'exprime également à travers des comportements encore plus traditionnels pour celles qui n'ont pas du tout travaillé avant la première maternité (traj. 5 avec 8,6\%). Ici, la dimension familiale prend davantage de place que la dimension professionnelle. Bien qu'elles aient terminé leurs études, on peut supposer qu'elles étaient alors faiblement diplômées et que la mise en couple était garante de leur sécurité financière (Sauvain-Dugerdil, 2005b). Plus nombreuses que les Suédoises à vivre cette trajectoire mais moins nombreuses que les Italiennes, il n'est pas possible de conclure que ces dernières présentaient un fort attachement pour le mariage étant donné le processus de mise en union assez varié dans ce régime durant la période observée. Tout de même, les transitions importantes de leur jeunesse sont consacrées à la fondation de leur famille tout en laissant de côté la dimension professionnelle.

Parallèlement, certaines ont eu une certaine diversité dans leur profil en choisissant des parcours avec une forte conciliation entre le travail et la famille (traj. 8 avec $8,5 \%)$. Celles-ci ont effectué leur passage à la vie adulte par l'emploi, tout en étant encore aux études au moment de la naissance de leur premier enfant. Ce cumul de plusieurs statuts en même temps semble être moins favorisé à travers le temps, puisque les plus jeunes en usent beaucoup moins que les plus âgées ont pu le faire ( $14 \%$ pour les plus âgées et $3 \%$ pour les plus jeunes).

Dans la même veine, certaines Françaises ont d'abord quitté leurs parents et ont choisi de vivre de manière autonome en finissant leurs études et en travaillant (traj. 4 
avec 7,7 \%). On peut toutefois supposer que la mise en couple s'est faite rapidement et a apporté un soutien non négligeable à la vie de ces femmes, compte tenu des tendances présentées concernant leurs trajectoires familiales/conjugales.

De façon générale, le modèle français se distingue par un mélange de stratégies essentiellement séquentielles, qui reflètent les aspects traditionalistes du régime conservateur, et de stratégies visant à concilier les différentes dimensions de la vie dans un cumul de plusieurs statuts. Ce modèle, qui persiste à travers le temps, permet de comprendre que si les Françaises se sont adaptées à la féminisation du marché du travail, elles ont conservé des traces de leurs réflexes traditionnels en adoptant une certaine sécurité dans l'enchaînement des évènements (Cavalli, 1993). Les trajectoires dominantes chez les Françaises ont très peu varié: les plus jeunes ont les mêmes probabilités que les plus âgées de ne pas travailler avant leur première maternité, avec respectivement $12,3 \%$ et $12,6 \%$ (traj. 1 et 5). Par contre, les plus jeunes tendent à se regrouper dans des trajectoires où elles auront terminé leurs études avant la première maternité, avec $66,5 \%$ pour les plus âgées contre $81,7 \%$ pour les plus jeunes (somme des traj. 3, 4, 5, 6, 7, 9 et 10).

\section{CONCLUSION ET DISCUSSION}

Durant les décennies observées, les parcours de vie des femmes vers la première maternité dans les trois régimes providentiels permettent de confirmer l'existence de modèles de cheminements distincts dans ces derniers avec un accès différent aux ressources requises pour avoir et élever un enfant (Majnomi-d'Intignano, 2004).

En Italie, le cheminement privilégié se concentre autour de l'institution du mariage : clé à travers le temps de la conciliation pour la création de la famille, phénomène qui rejoint la caractéristique principale du régime familialiste axé sur l'aide de la famille (Mathieu, 2003). L'institution prime, durant toute la durée observée, sur le choix de trajectoires alternatives, dans la mesure où les jeunes Italiennes retardent leur entrée dans la vie adulte pour pouvoir vivre la trajectoire «traditionnelle» (Livi Bacci et al., 2000). La conciliation dans la mobilisation des ressources scolaires/professionnelles conserve également son aspect traditionnel. Certaines Italiennes présentent un parcours axé sur la répartition des rôles de genre, où la mère demeure à la maison et n'aura jamais travaillé avant d'enfanter, et d'autres intègrent l'emploi, mais choisissent tout de même de vivre la trajectoire traditionnelle type qui correspond à l'enchaînement suivant: «fin des études», «premier emploi», «mise en couple» puis «premier enfant» (Cavalli et al., 1993). Les deux types de trajectoires courantes chez les Italiennes misent sur une mobilisation sécuritaire des ressources avant la première maternité: la première met en relief l'importance du mari comme apport principal au revenu de la famille et la deuxième correspond à une trajectoire assez traditionnelle, qui présente une mobilisation séquentielle des ressources (les deux grandes dimensions de la vie des femmes sont vécues l'une après l'autre). Ces modèles de trajectoire ont persisté à travers le temps. L'augmentation de la participation des femmes au marché du travail italien, même si elle demeurait faible, illustre toutefois la préférence des plus jeunes 
Italiennes pour la trajectoire de type séquentiel, qui sépare les différentes dimensions dans le parcours de vie (Njoki, 2006).

En Suède, les cheminements reflètent fortement le contexte social-démocrate que décrit la littérature. En effet, le modèle traditionnel n'y est pratiquement plus vécu et la variété des trajectoires alternatives choisies illustre le développement de multiples possibilités de vie pour les Suédoises (Croisetière, 2006). Elles accentuent d'ailleurs à travers le temps cette variété de comportements à tel point d'ailleurs qu'il devient difficile de tenir compte de l'ensemble des évènements vécus par les Suédoises avant leur première maternité: elles peuvent vivre des mises en union successives, et/ou de différente nature, tout en les faisant précéder fréquemment d'une période de célibat. Quant au modèle suédois de cheminement scolaire/professionnel, il porte aussi les traces du régime social-démocrate. Les Suédoises se démarquent par un éclatement important au sein de plusieurs trajectoires alternatives qui cumulent plusieurs statuts. Ce modèle s'est par ailleurs accentué à travers le temps, essentiellement dans le nombre de séquences vécues. Les plus jeunes Suédoises ont vécu plusieurs évènements leur permettant d'expérimenter un certain nombre de statuts avant de vivre une première maternité (Groulx, 1990; Pinelli et al., 2001).

En France, le cheminement des femmes se démarque par un modèle hybride, qui se situe entre les trajectoires éclatées des Suédoises et la forte concentration des Italiennes autour de l'événement unique du mariage. En fait, ce sont essentiellement les plus jeunes qui présentent des caractéristiques similaires aux Suédoises: préférence pour la cohabitation hors mariage, les unions successives ou encore une période préalable de célibat. Au contraire, les Françaises plus âgées, à l'image des Italiennes, conservent des comportements plus traditionnels, favorisant l'événement central du mariage. En outre, si certaines Françaises participent rapidement au marché du travail et choisissent de vivre des trajectoires qui s'appuient sur un cumul des statuts et une conciliation travail/famille, encore nombreuses sont celles qui privilégient la trajectoire traditionnelle pour ses aspects sécuritaire et séquentiel. Contrairement au modèle de trajectoire familiale/conjugale, la trajectoire scolaire/professionnelle évolue toutefois peu à travers le temps (Ekert-Jaffé et al., 2002).

En conclusion, les différents régimes influent sur les parcours de vie et contraignent les individus à ajuster leur comportement en faveur ou en défaveur de leur projet de vie, choisissant les parcours qui leur permettront de créer une famille dans les conditions idéales. Les modèles d'États providentiels fortement ancrés depuis plusieurs décennies montrent qu'ils ont une capacité de résilience importante, notamment dans les parcours de vie (McDonald, 1997). Il semble dès lors intéressant de noter que le régime social-démocrate illustre fort bien les propos de McDonald qui, en voulant réconcilier les féministes et les natalistes, souligne qu'il existe une forte relation entre la promotion de l'égalité entre hommes et femmes et le fait d'avoir une descendance finale plus importante et nous rajouterons des parcours de vie conciliants. 


\section{BIBLIOGRAPHIE}

Аввотт, А. (1995), «Sequence Analysis: New Methods for Olds Ideas», Annual Review of Sociology, vol. 21, p. 93-113.

Bernard, P. (2007), «Les dynamiques inter-reliées des processus liés aux variations démographiques et aux parcours de vie», Horizons: Projet de recherche sur les politiques, vol. 9 (4), p. 13-16.

Billari, F. C. (2006), «Timing, Sequencing, and Quantum of Life Course Events: A Machine Learning Approach», European Journal of Population, vol. 22, p. 37-65.

Billari, F. C. (2004), «Becoming an Adult in Europe: A Macro (/Micro)-Demographic Perspective», Demographic Research, Special collection, vol. 3 (2).

Brachet, S. (2001), Politique familiale et assurance parentale en Suède: une synthèse. Dossier d'études, no $21, \mathrm{CNAF}$.

Cassese, S. (2001), Portrait de l'Italie actuelle, Études de la Documentation Française.

Cavalli, S. (2003). «Le parcours de vie: entre institutionnalisation et individualisation», L'avenir. Attentes, projets, (dés)illusions, ouvertures, Lausanne, Éditions Réalités sociales.

Cavalli, A. et O. Galland (1993), L'allongement de la jeunesse. Le changement social en Europe occidentale, Arles, Actes Sud.

Charton L. (2003), Calendriers familiaux et rapport au temps: la diversification des comportements et des parcours familiaux en Europe. Thèse de doctorat présenté à Strasbourg, Université Marc Bloch, France.

Cicchelli, V. et M. Merico (2007), «Le passage tardif à l'âge adulte des Italiens: entre maintien du modèle traditionnel et individualisation des trajectoires biographiques », Horizons Stratégiques, Revue trimestrielle du Centre d'analyse stratégique, $\mathrm{n}^{\circ} 4$.

Cohu, S., M. Lelièvre, D. Lequet-Slama et O. Thévénon (2005), «Les politiques en faveur de la famille: de nouveaux enjeux pour les pays d'Europe du Sud», Études et Résultats, n 449, Direction de la recherché des études de l'évaluation et des statistiques, Dreess.

Cooke, L. P. (2006), «Le Sud revisité: équité de genres et fécondité en Italie et en Espagne», Recherches et Prévisions, Dossier comparaisons internationales, $\mathrm{n}^{\circ} 83$, p. 61-77.

Courgeau, D. et E. Lelièvre (1989), Analyse démographique des biographies. Éditions de l'institut national d'études démographiques, p. 269.

Croisetière, P. (2006), "Portraits des systèmes éducatifs et de garde dans neuf pays développés ", Recherche et Rédaction, Ministère de la Famille, des Aînés et de la Condition féminine, Édition Direction des relations publiques et des communications.

Dufour-Kippelen, S. (2001), «Les déterminants des trajectoires de décohabitation et d'accès à l'emploi des jeunes peu diplômés», Recherches et Prévisions, Dossier jeunes adultes, $\mathrm{n}^{\circ} 65$.

Elder, G. H. (1998), "The life Course and Human Development», in R.M. Lerner (dir.). Handbook of Child Psychology. Volume 1: Theorical Models of Human Development, New York, Wiley \& Sons, p. 939-991.

Ekert-Jaffé, O., H. Joshi, K. Lynch, R. Mougin et M. Rendall (2002), «Fécondité, calendrier des naissances et milieu social en France et en Grande-Bretagne: Politiques sociales et polarisation socioprofessionnelle», Population, 57 (3), p. 485-518.

Esping-Andersen, G. (1999), Les trois mondes de l'État-providence, Paris, PUF.

Festy, P. et F. Prioux (2002), An evaluation of the Fertility and Family Surveys Project, Institut national d'études, United Nations Economic Commission for Europe.

Galland, O. (2000), "Entrer dans la vie adulte: des étapes toujours plus tardives mais resserrées», Économie et Statistique, $\mathrm{n}^{\circ} 337-338$, p. 7-8.

Galland, O. et B. Roudet (2005), Les jeunes Européens et leurs valeurs, Paris, La Découverte.

Gauthier, A. H. (2002), «Les politiques familiales dans les pays industrialisés: y a-t-il convergence?», Population, vol. 53 (3), p. 457-484.

Groulx, L.-H. (1990), Où va le modèle suédois? État providence et protection sociale. Presse de l'Université de Montréal, Édition l'Harmattan. 
Livi Bacci, M. et S. SAlvini (2000), «Trop de famille et trop peu d'enfants: la fécondité en Italie depuis 1960 », Cahier Québécois de Démographie, vol.49 (2), p. 231-254.

Majnoni d'Intignano, B. (2004), «Travail féminin et différences de fécondité en Europe», Population et Avenir, (juin).

Malpas, N. (1999), «Les couples européens: qui sont-ils?», Cahier québécois de démographie. vol. 28 (1-2), p. 117-149.

Marc, C. et H. Zajdela (2005), Emploi des mères et politique familiale: doit-on s'inspirer du «modèle suédois»? Centre d'études de l'emploi, Matisse, $\mathrm{n}^{\circ} 45$.

Mатнieu, S. (2003), Droits, marchandisation et défamilialisation: une typologie des régimes de genre, Mémoire de maitrise, Département de sociologie, Université de Montréal.

McDonald, P. (1997), «Gender Equity, Social Institutions and the Future of Fertility» in M. E. CosioZavala (dir.)Women and Families: Evolution of the Status of Women as Factor and Consequence of Changes in Family Dynamics, Paris, Cicred, p. 13-33.

Monnier, A. (2006), Démographie contemporaine de l'Europe: évolutions, tendances, défis, Paris, Armand Colin.

McDonald, P. (2002), «Les politiques de soutien de la fécondité: l'Éventail des possibilités», Population, $57^{\mathrm{e}}$ Année, (3), p. 423-456.

Munoz-Perez, F. (1986), «Changements récents de la fécondité en Europe occidentale et nouveaux traits de la formation des familles», Population, vol. 41 (3), p. 447-462.

Nicole-Drancourt, C. et L. Roulleau-Berger (2006), L'insertion des jeunes en France, Paris, PUF, Que sais-je?, p. 126.

Njoki Friedli, M. (2006), L'influence de la politique familiale sur la fécondité des femmes en Italie, France, Suède et en Suisse: une étude comparative, Mémoire de Maîtrise, Université de Genève.

Palomba, R. (2001), Postponement in Family Formation in Italy, within the Southern European Context, Paper presented at the IUSSP Seminar on International Perspectives on Low Fertility Trends, Theories and Policies, Tokyo, Japan, 21-23 March.

Pauti, A. (1992), «La politique familiale en Suède», Population, $47^{e}$ année (4), p. 961-985.

Pinelli, A. et al. (2001), «Fécondité et nouveaux types de ménages et de formation de la famille en Europe», Études démographiques, n 35, Édition Conseil de l'Europe.

Rajulton, F. (1999), LIFEHIST: Analysis of Life Histories, a State Space Approach, Paper presented at the workshop on Longitudinal Research in Social Science: A Canadian Focus, London, Ontario.

Sauvain-Dugerdil, C. (2005b), «La place de l'enfant dans les projets de vie: temporalité et ambivalence», chapitre 7 dans: Maternité et parcours de vie, Michel Oris (dir.), vol 4., p. 281-316.

Toulemon, L. (1994), «La place des enfants dans l'histoire des couples», Population, vol. 49 (6), p. 13211345. 